\title{
The methylation of SDC2 and TFPI2 defined three methylator phenotypes of colorectal cancer
}

\author{
Ruixue Lei ${ }^{1^{*}}$, Yanteng Zhao ${ }^{2 *}$, Kai Huang ${ }^{3}$, Kangkang Wan ${ }^{4}$, Tingting $\mathrm{Li}^{4}$, Haijun Yang ${ }^{1{ }^{* *}}$, \\ Xianping $\mathrm{Lv}^{2 * *}$ \\ ${ }^{1}$ Department of Pathology, The Fourth Affiliated Hospital of Henan University of \\ Science and Technology, Anyang Tumor Hospital, Anyang, Henan, China \\ ${ }^{2}$ Department of Transfusion, The First Affiliated Hospital of Zhengzhou University, \\ Zhengzhou 450052, China \\ ${ }^{3}$ Department of Gastrointestinal Surgery, Department of General Surgery, the First \\ Affiliated Hospital of Anhui Medical University, Hefei, China \\ ${ }^{4}$ Wuhan Ammunition Life-tech Company, Ltd., Wuhan, Hubei, China \\ *Contributed equally. \\ ** Co-corresponding authors.
}

Corresponding authors:

Haijun Yang

yhj1972@126.com

Xianping Lv

Ivxianping@126.com

\begin{abstract}
Methylation-based noninvasive molecular diagnostics are easy and feasible tools for the early detection of colorectal cancer (CRC). However, many of them have the limitation of low sensitivity with some CRCs detection failed in clinical practice. In this study, the clinical and pathological characteristics, as well as molecular features of three methylator-groups, defined by the promoter methylation status of SDC2 and TFPI2, were investigated in order to improve the performance of CRC detection. The Illumina Infinium 450k Human DNA methylation data and clinical information of CRCs were collected from The Cancer Genome Atlas (TCGA) project and Gene Expression Omnibus (GEO) database. CRC samples were divided into three groups, HH (dual-positive), HL (single positive) and LL (dual-negative) according to the methylation status of SDC2 and TFPI2 promoters. Differences in age, tumor location, microsatellite instable status and differentially expressed genes (DEGs) were evaluated among the three groups and these findings were then confirmed in our inner CRC dataset. The combination of methylated SDC2 and TFPI2 showed a superior performance of distinguishing CRCs from normal controls than each alone. Samples of HL group were more often originated from left-side CRCs whereas very few of them were from right-side $(P<0.05)$. HH grouped $\mathrm{CRCs}$ showed a higher level of microsatellite instability and mutation load than other two groups (mean nonsynonymous mutations for
\end{abstract}


HH/HL/LL: $10.55 / 3.91 / 7.02, P=0.0055)$. All mutations of $B R A F$, one of the five typical CpG island methylator phenotype (CIMP) related genes, were found in $\mathrm{HH}$ group $(\mathrm{HH} / \mathrm{HL} / \mathrm{LL}: 51 / 0 / 0, P=$ 0.018). Also there was a significantly older patient age at the diagnosis in $\mathrm{HH}$ group. Gene expression analysis identified 37, 84 and 22 group-specific DEGs for HH, HL and LL, respectively. Functional enrichment analysis suggested that HH specific DEGs were mainly related to the regulation of transcription and other processes, while LL specific DEGs were enriched in the biological processes of extracellular matrix interaction and cell migration. The three defined mathylator groups showed great difference in tumor location, patient age, MSI and ECM biological process, which could facilitate the development of more effective biomarkers for CRC detection.

Key words: colorectal cancer, early detection, methylator phenotypes, SDC2, TFPI2, biomarker

\section{Introduction}

Colorectal cancer (CRC) is responsible for over 1 million new cases every year and around 700,000 deaths occurred worldwide, making it the third most frequently diagnosed cancer [1,2]. In China, the incidence and mortality of CRC has been witnessed with an increasing trend of 12.8 in 2003 to 16.8 per 100,000 in 2011 and 5.9 in 2003 to 7.8 per 100,000 in 2011, respectively [3]. It is believed that CRCs represent a heterogeneous group of tumors characterized by complex multifactorial phenotypes and no single risk factor is responsible for the developing of CRC $[4,5]$. Many factors including diet, tobacco smoking, microbial, overweight and obesity, genetic factors, as well as metabolic and other exposures can alter the risk of getting CRC [6-9]. Nearly half of colorectal cancer incidence and mortality was attributable to unhealthy diets such as low vegetable and fruit intake, high red and processed meat intake, and alcohol drinking etc., in China in 2012 [8]

Syndecan-2 (SDC2), as one of the syndecan family of heparan sulfate proteoglycan, has been demonstrated playing an important role in cancer progression through regulation of cell adhesion, proliferation, and migration in many researches [10-13]. Tissue factor pathway inhibitor-2 (TFPI-2), belongs to the Kunitz-type serine proteinase inhibitor family and is thought to be functional in the regulation of extracellular matrix digestion and re-modeling by inhibiting a broad spectrum of serine proteinases $[14,15]$. Unlike the tumorigenic behaviors of $S D C 2$ in colon cancer cells, TFPI2 has been shown as a tumor suppressor gene in several malignant tumors [16-19]. However, both promoters of the two genes were found with frequently hyper-methylated status in colon cancer cells compared to normal tissue cells in a few epi-genomics studies $[20,21]$. The frequently aberrant DNA methylation of SDC2 and TFPI2 makes them promise biomarkers for the early detection of CRC [22-24] and hence were also used as CRCs diagnostic biomarkers in our preliminary clinical trials. Molecular subtyping analysis based on DNA methylomics data identified a subset of CRCs, CpG island methylator phenotype (CIMP), which is characterized by significant hyper-methylated $\mathrm{CPG}$ islands of tumor suppressors [25]. When combined with the clinicopathological parameters and molecular characteristics, CIMP tumors showed significant associations with BRAF mutations, MSI-H, female sex, right-sided tumor location, and age [25]. Additionally, prognostic analysis showed that CIMP-high patients presented a worse prognosis than CIMP-low, which suggested CIMP could be a predictor of 
prognosis of CRC [26].

CRC is a disease with high heterogeneity and many differences were observed among CRCs raised from proximal (right) or distal (left) colon. For example, right-sided colon cancers were reported an increased incidence of proximal migration, while it was inversed for rectosigmoid tumors [27]. What's more, the incidence rates between proximal colon and distal colon also differ in age and gender [28]. These data reflect an extensively distinct molecular pathogenesis between the cancers originated from these two anatomical locations, which might be related to significant impact on tumorigenesis in these respective sides [28]. In terms of genomic features, proximal carcinomas are characterized by more often microsatellite instable (MSI), frequent BRAF-mutated and expressing the CIMP phenotype $[29,30]$. Therefore, tumor location would be an important factor of biological heterogeneity and it should be reasonable to group CRC into right-sided (proximal) and left sided (distal) ones [31].

Several studies have suggested a better performance of combined multi-targets for CRC early detection than single biomarker [32-34]. However, during clinical practice, some CRC samples was detected with only single or no target positively, reflecting the preference of different targets in distinguishing CRCs from normal samples. In this study, we first classified CRC samples into three methylator groups, SDC2/TFPI2 double positive group $(\mathrm{HH}$, Hypermethylation-hypermethylation), SDC2/TFPI2 single positive group (HL, hypermethylation-hypomethylation) and SDC2/TFPI2 double negative group (LL, hypomethylation-hypomethylation) according to the promoter methylation status of $S D C 2$ and TFPI2, which were previously determined as dual-targets for CRC early detection by our custom window-sliding method (data not published). The clinicalpathological parameters and molecular features were then evaluated by inner and outer samples including TCGA COAD/READ cohorts, GEO datasets as well as our D311 CRC dataset. These findings indicated that it might be reasonable to define three SDC2/TFPI2 methylator groups according to their methylation status and will benefit the development of more effective methylated biomarkers.

\section{Methods}

\section{Data preparation}

The level 3 methylation data, raw read-count of RNA-seq and clinical information of colon adenocarcinoma and rectum adenocarcinoma patients were retrieved from The Cancer Genome Atlas (TCGA) data portal (https://portal.gdc.cancer.gov/) by using the TCGAbiolinks R package [35]. The platform of methylation data from TCGA is Illumina Human Methylation 450 Beadchip (450K array) and we also searched the Gene Expression Omnibus (GEO; http://www.ncbi.nlm.nih.gov/geo/) for eligible datasets that are generated by $450 \mathrm{~K}$ array. Two GEO datasets, GSE48684 [36] and GSE79740 [37], were then downloaded because of their available clinical information.

Empirical Bayes (EB) batch adjustment along with two step quantile normalization method [38] was conducted for batch effect removal before GSE48684 and GSE79740 datasets were merged as one set. Missing values of the $450 \mathrm{k}$ array were inferred and fulfilled by the Bayesian Network 
structure learning algorithm [39]. All samples without clinical information were removed and the information of preprocessed data used in this study was presented in Table 1.

\section{Patient samples}

Fresh-frozen colorectal cancer tissues $(n=300)$ and colorectal mucosa $(n=55)$ tissues were collected at Zhongnan Hospital of Wuhan University at the time of surgery. All objects recruited signed a written informed consent and their final diagnosis were determined based on colonoscopy or histological test. Participants who undertook any chemotherapy or radiotherapy, or had incomplete information were excluded. The collected information consist of age, gender, tumor size, tumor location, grade and MSI status. Detailed demographic and clinical features of the subjects were listed in Table 1 . We defined rectosigmoid, descending colon, and splenic flexure tumors as left-sided cancer, whereas hepatic flexure and ascending colon tumors were grouped as right-sided cancer [40]. This study was approved by the medical ethics committee of Zhongnan Hospital of Wuhan University (No.2019099).

Table 1 Clinical characteristics of subjects.

\begin{tabular}{lccc}
\hline Subject characteristics & TCGA CRC & GSE48684/GSE79740 & D311 CRC \\
\hline Normal & 45 & 51 & 54 \\
Tumor & 394 & 108 & 257 \\
Age,no.(\%) & & & \\
$>=60$ & $254(64.96 \%)$ & Not available & $149(58.75 \%)$ \\
$<60$ & $137(35.04 \%)$ & Not available & $106(41.25 \%)$ \\
Localization, no. (\%) & & & \\
Left Colon & $148(37.56 \%)$ & $72(66.67 \%)$ & $82(31.91 \%)$ \\
Right Colon & $147(37.31 \%)$ & $28(25.93 \%)$ & $49(19.07 \%)$ \\
Rectum & $46(11.68 \%)$ & $6(5.56 \%)$ & $90(35.02 \%)$ \\
Others & $53(13.45 \%)$ & $2(1.85 \%)$ & $36(14.01 \%)$ \\
Gender,no.(\%) & & & \\
Male & $211(53.55 \%)$ & Not available & $152(59.14 \%)$ \\
Female & $183(46.45 \%)$ & Not available & $105(40.86 \%)$ \\
MSI-H,no.(\%) & $65(16.75 \%)$ & Not available & $15(8.06 \%)$ \\
MSS,no.(\%) & $323(83.25 \%)$ & Not available & $171(91.94 \%)$ \\
\hline
\end{tabular}

\section{Methylation-specific PCR experiments}

The genomic DNA were extracted by UnigeneDx FFEE DNA extraction kit according to manufacturer's instruction. Target genes in tumor tissue were captured using previously reported technology with some modification [24]. Tissue derived genomic DNA was chemically modified by sodium bisulfite to convert unmethylated cytosine to uracil while leaving methylated cytosine unchanged. Methylation-specific PCR (MSP) was used to determine the methylation status of SDC2 and TFPI2 in normal and tumor tissue DNA, $\beta$-actin [41] was used as internal control. Specific primers and probe for the target region of SDC2 and TFPI2 was designed as showing in 
Table 2. We use the cycle threshold $(\mathrm{Ct})$ value to determine the methylation status of these two genes and the values for tissue samples were considered "invalid" if the ACTB Ct was greater than 36.00 and methylated SDC2/TFPI2 were considered "detected" if the Ct values were less than 45.00. For samples with no amplification curve of the MSP occurred after 45 cycles, Ct value was assigned 45.00. Three MSP replicates was done for each sample and the average $\mathrm{Ct}$ value was used for further analysis.

Table 2 Primers and probes used in this study

\begin{tabular}{lllc}
\hline Name & Primer/Probe sequences $\left(5^{\prime}\right.$ - 3') $^{\prime}$ & Description & Length \\
\hline SDC2_F & CGAGTTGAGTCGTAATCGTTGC & MSP region 1 forward primer & \\
SDC2_R & TCCGCCGACACGCAAACCACCAAACC & MSP region 1 reverse primer & 187 bp \\
SDC2_P & AACAAAACGAAACCTCCTACCCAAC & MSP region 1 probe & \\
TFPI2_F & CGCGGAGATTGTITITGT & MSP region 2 forward primer & \\
TFPI2_R & AACAAACATCGTCGCAAACCTC & MSP region 2 reverse primer & 163 bp \\
TFPI2_P & ATAAAACCCGACAAAATCCG & MSP region 2 probe & \\
ACTB_F & CGCAATAAATCTAAACAAACTCC & ACTB forward primer & \\
ACTB_R & AGGTTAGATGGGGGATATGT & ACTB reverse primer & 89 bp \\
ACTB_P & TCCCAAAACCCCAACACACT & ACTB probe & \\
\hline
\end{tabular}

\section{Identification of differentially expressed genes}

Level 3 RNA-seq data of TCGA CRCs was preprocessed before differentially expressed analysis by removing low expressed genes whose expressions were zero among more than $90 \%$ samples. DESeq2 [42] (V1.30.0) package was used to perform a pairwise comparison between all the three methylator groups for the identification of differentially expressed genes (DEGs). Adjusted $P$ values were calculated by false discovery rate (FDR) method. Genes with adjusted $P$ value $<0.05$ were selected as DEGs and used for further functional enrichment analysis. We used GeneCodis [43] for the enrichment analysis of GO biology process (BP) and KEGG pathway for identified

DEGs。

\section{Statistical analysis}

Comparisons for two paired or unpaired samples were performed for continuous variables using paired or unpaired student t-test. Kruskal-Wallis nonparametric analysis were used for multi-group comparisons of continuous variables. For categorical variables, fisher's exact test was applied to determine if there are nonrandom associations between SDC2/TFPI2 methylator groups and clinical characteristics, such as age, sex and tumor location. For $450 \mathrm{k}$ array data, it was proposed to use the thresholds of 0.2 and 0.8 to define hypo- and hyper-methylation $[44,45]$. In this study, we grouped CPG sites into two categories, hypo-methylated and hyper-methylated based on their $\beta$ values with the threshold of 0.2. Our D311 CRC samples are defined as detectable negative if $\mathrm{Ct}$ values of both the dual-targets are $>38$, otherwise they are defined as detectable positive. Sensitivity and specificity were calculated as follows: 


$$
\begin{aligned}
& \text { Sensitivity }(\%)=\text { true positives/total number of CRC patients * } 100 \\
& \text { Specificity }(\%)=\text { true negatives/total number of controls * } 100
\end{aligned}
$$

The performance of the diagnostic models were evaluated by the area under receiver operating characteristic (ROC) curve (AUC) and $95 \%$ confidence interval (CI). All statistical analyses were performed using R software (version 3.6.0).

\section{Results}

\section{The methylation of SDC2 and TFPI2 showed good discrimination of CRCs from normal controls}

Probes within 2 kilo-base upstream of the transcription start site of SDC2 and TFPI2 were selected and their mean methylation levels were then calculated according to the $\beta$ values of $450 \mathrm{k}$ array by using TCGA and GEO CRC samples. We only used the probes with $\Delta \beta$ ( $\beta$ Tumor- $\beta$ Normal) $>=0.3$ and identified 4 and 7 probes in the promoter of SDC2 and TFPI2 (Table 3). The average $\beta$ values of these filtered probes was used as the methylation level of SDC2 and TFPI2 (herein termed as SDC2_P and TFPI2_P). A lower $\triangle \beta$ value was found for TFPI2 than SDC2 which might attribute to its higher background methylation level on normal controls (Figure 1A). We also observed a higher sensitivity and lower specificity for TFPI2 than SDC2 (Figure 1B). When combining SDC2 and TFPI2, the diagnostic sensitivity was improved (Figure 1B), which demonstrated that dual-target biomarkers could distinguish CRCs from normal controls better than single target. In our clinical outcomes, CRCs showed significantly higher methylation levels than normal (Figure 1C). The pattern of specificity and sensitivity, as well as the combined sensitivity for SDC2 and TFPI2, were also in line with our former results.

Table 3 Probes identified in the promoter of SDC2 and TFPI2.

\begin{tabular}{cllll}
\hline Symbol & Probes & Mean $\beta_{N}(s d)$ & Mean $\beta_{T}(s d)$ & $\Delta \beta$ \\
\hline SDC2 & cg16935295 & $0.027(0.015)$ & $0.580(0.231)$ & 0.553 \\
& cg04261408 & $0.136(0.058)$ & $0.789(0.197)$ & 0.653 \\
& $\operatorname{cg} 14625631$ & $0.065(0.013)$ & $0.327(0.183)$ & 0.262 \\
& $\operatorname{cg} 10292139$ & $0.073(0.034)$ & $0.609(0.271)$ & 0.537 \\
TFPI2 & $\operatorname{cg} 12973591$ & $0.163(0.097)$ & $0.641(0.160)$ & 0.478 \\
& $\operatorname{cg} 14377593$ & $0.145(0.069)$ & $0.506(0.172)$ & 0.361 \\
& $\operatorname{cg} 17338208$ & $0.183(0.120)$ & $0.645(0.190)$ & 0.462 \\
& $\operatorname{cg} 22441533$ & $0.058(0.013)$ & $0.233(0.161)$ & 0.176 \\
& $\operatorname{cg} 22799321$ & $0.208(0.085)$ & $0.650(0.182)$ & 0.441 \\
& $\operatorname{cg} 24531255$ & $0.246(0.117)$ & $0.594(0.155)$ & 0.348 \\
SDC2_P & $\operatorname{cg} 26739865$ & $0.175(0.070)$ & $0.621(0.197)$ & 0.446 \\
TFPI2_P & & $0.074(0.023)$ & $0.562(0.188)$ & 0.488 \\
\hline
\end{tabular}

$\Delta \beta=\beta_{T}-\beta_{N}$ 


\section{HL group CRCs were more likely originated from left-side colon}

We defined three methylator groups, $\mathrm{HH}$ (dual-positive), $\mathrm{HL}$ (single positive) and $\mathrm{LL}$ (dual-negative) according the promoter methylation status of SDC2 and TFPI2 (see methods). During the development of diagnostic biomarkers, CRCs divided to HL and LL groups are very important because of their effect on the sensitivity which might reflect a preference of the biomarkers for certain CRC subgroups. We first compared the three methylator groups with tumor location, and found that HL group CRCs were more frequently originated from left-side colon. A small amount of CRCs were from rectum, however very few were from right-side colon (Figure 2A-C, Table 4). This result indicated a potential impact of tumor locations on the early detection of CRCs by using SDC2/TFPI2 dual-targets.

Table 4 Samples in three methylator groups compared with tumor locations

\begin{tabular}{lcccccccccc}
\hline \multirow{2}{*}{ Tumor location } & \multicolumn{3}{c}{ TCGA CRC } & \multicolumn{3}{c}{ GSE48684/GSE79740 } & \multicolumn{3}{c}{ D311 } \\
\cline { 2 - 11 } & HH & HL & LL & HH & HL & LL & HH & HL & LL \\
\hline Right-side & 143 & 2 & 2 & 26 & 0 & 2 & 47 & 1 & 1 \\
Left-side & 120 & 25 & 3 & 66 & 5 & 1 & 65 & 12 & 3 \\
Rectum & 41 & 4 & 1 & 4 & 2 & 0 & 73 & 14 & 4 \\
Other & 49 & 3 & 1 & 1 & 1 & 0 & 34 & 3 & 0 \\
$X^{2} P$ & 0.00059 & & & 0.015 & & & 0.081 & \\
\hline
\end{tabular}

\section{HH group CRCs presented more frequently genomic instability}

Microsatellite instability and hypermutation have been regarded as important molecular characteristics of CRCs. Compared to other two groups, HH group CRCs presented the highest mutation load (Figure 3A, P < 0.05) in TCGA CRC dataset. By using the MANTIS score [46], which is used to evaluate the MSI status, we grouped the TCGA CRCs into MSI-H and MSS if this score is > 0.4 . The $\beta$ values of SDC2 and TFPI2 showed a high concordance with MANTIS score in MSI-H group (Figure 3B, $\mathrm{P}<0.001$ ), which is consistent with the result that higher mutation load occurred in $\mathrm{HH}$ group. We studied the association of three methylator groups with the mutation of 5 typical CIMP-related genes including BRAF, PIK3CA, KRAS, TP53 and APC. All BRAF mutated $\mathrm{CRCs}$ were in $\mathrm{HH}$ group (Figure $3 \mathrm{C}, \mathrm{HH} / \mathrm{HL} / \mathrm{LL}: 51 / 0 / 1, \mathrm{P}=0.018$ ). We further compared the association between MSI-status and tumor locations with TCGA CRCs and our D3111 CRCs. The MSI-H CRCs were more preference in the right-side colon (Figure 3C\&D, $P<0.001$ ), which possibly elucidated potential causal factors that $\mathrm{HL}$ group CRCs were mainly in left-side colon and MSI-H CRCs were less likely in HL group.

\section{Older patients age was found in $\mathrm{HH}$ group CRCs}

The age of patients is one of the risk factor for colon cancer and we found a significantly older age in $\mathrm{HH}$ group patients, while it was the youngest for LL group patients (Figure $4 A \& B, P<0.05$ ). Since the genomic DNA methylation is associated with patient age, we observed a positive 
correlation of the methylation levels of SDC2_P and TFPI2_P with patient age (Figure 4C\&D), indicating that young patients might be more likely to be undetected.

\section{LL group CRCs might be related to alterations of ECM and cell migration biological}

\section{processes}

We performed differential expression analysis by using the gene expression profile of TCGA CRCs to identify group-specific DEGs. A total of $37 \mathrm{HH}$ specific, $84 \mathrm{HL}$ specific and $22 \mathrm{LL}$ specific DEGs were identified according to their average expression values on the three groups (Figure 5A). Functional enrichment analysis implied that $\mathrm{HH}$ specific DEGs were mainly related to the regulation of transcription and other processes (Figure 5B), while LL specific DEGs are enriched in the biological processes of extracellular matrix interaction (ECM) and cell migration (Figure 5C). These results might elucidate potential alterations in the biological processes of ECM and cell migration that lead to different characteristics of these three groups.

\section{Discussion}

Quantifying aberrant methylated genes was useful and feasible method for the early detection of CRCs. However many of these biomarkers have the limitation of low sensitivity such as the undetected false negative samples [24,47]. In this study, we defined three CRC methylator groups, dual-positive $(\mathrm{HH})$, single positive $(\mathrm{HL})$ and dual-negative (LL) based on the methylation status of SDC2 and TFPI2 and then evaluated their characteristics of genomic instability, mutation load, patient age, and biological processes.

The performance of SDC2 and TFPI2 for discriminating CRCs from normal controls was first evaluated in three independent datasets and our preliminary study revealed a relatively higher specificity and lower sensitivity for detection of SDC2 methylation than TFPI2. This might be attributed to the higher background methylation level in the promoter of TFPI2. The detected sensitivity was improved when combing the dual-targets compared to single target, indicating a complemental performance of SDC2 and TFPI2 in early detection of CRCs. Previous studies have demonstrated that multi-target outperformed single target $[48,49]$, which was also found in this study.

In clinical practice, the single-positive and dual-negative CRCs detected by dual-target will cut down the sensitivity of these biomarkers: Our findings indicated that single-positive CRCs more likely tend to originate from left-side colon. Several studies have demonstrated that left-side colon presents lower degrees of methylation, while right-side colon shows high degrees of methylation which was called the $C P G$ island methylator phenotype, or CIMP [50]. This might explain why single-positive CRCs appear more frequently in left-side colon. Additionally, we found a positive correlation of the methylation levels of SDC2 and TFPI2 with MSI scroe in MSI-H CRCs, as well as lower mutation load and rare BRFA mutations in HL group CRCs. These results, on the other hand, confirmed that the molecular events, such as epigenetic instability, aberrant DNA 
mutation and MSI, are coupled with each other [25]. Gene expression analysis identified methylator group-specific DEGs and functional annotation of LL-specific DEGs was suggested to focus on the biological process of ECM-receptor interaction implying a potential alteration of molecular pathway in LL group CRCs. Interestingly, many studies have showed very important roles of SDC2 wan TFPI2 in the interaction of extracellular matrix with cell plasma [15,51]. These findings revealed the possible impact of ECM process on the performance of SDC2/TFPI2 dual-target in detecting CRCs.

Colorectal cancer is a disease with high heterogeneity. CRCs are often classified to proximal (right side) and distal (left-side) according to their anatomical locations. This classification is reasonable because of their distinctive embryonic derivation, which is the midgut and the hindgut for the proximal and distal colon, respectively $[27,31]$. It might be related to the differences in DNA methylation between left- and right-side colon, with potential impact on the detection of CRCs based on methylation status. In summary, the current study demonstrated the possible association of CIMP phenotype, tumor location and MSI with the dual-target in CRC early diagnosis. Our observations also suggested that it should be considered during the development of new methylation based biomarkers for CRC detection in these respective sides.

\section{Acknowledgments}

This study was supported by Medical Science and Technology Research Plan Joint Construction Project of Henan Province (Granted No. 2018020121). We declare that submitted manuscript and other materials are not under consideration for publication elsewhere. All authors listed have read the complete manuscript and have approved submission of the paper.

\section{Conflict of Interest}

All authors declare no conflict of interest.

\section{Data Availability Statement}

The TCGA CRC 450k data and GEO datasets are publicly available online. The D311 data used and analyzed in study are available from the corresponding author on reasonable request.

\section{Author Contributions}

Study design: HJ Y.

Data collection and analysis: RX L, YT Z, KK W and TT L. 
bioRxiv preprint doi: https://doi.org/10.1101/2021.03.03.433833; this version posted March 6, 2021. The copyright holder for this preprint (which was not certified by peer review) is the author/funder, who has granted bioRxiv a license to display the preprint in perpetuity. It is made available under aCC-BY-NC-ND 4.0 International license.

Manuscript writing: RX L, YT Z, K H and KK W.

Manuscript and results revising: $H J Y$ and $X P L$. 
bioRxiv preprint doi: https://doi.org/10.1101/2021.03.03.433833; this version posted March 6, 2021. The copyright holder for this preprint (which was not certified by peer review) is the author/funder, who has granted bioRxiv a license to display the preprint in perpetuity. It is made available under aCC-BY-NC-ND 4.0 International license.

\section{Figures}


bioRxiv preprint doi: https://doi.org/10.1101/2021.03.03.433833; this version posted March 6, 2021. The copyright holder for this preprint (which was not certified by peer review) is the author/funder, who has granted bioRxiv a license to display the preprint in perpetuity. It is made available under aCC-BY-NC-ND 4.0 International license.

A

B
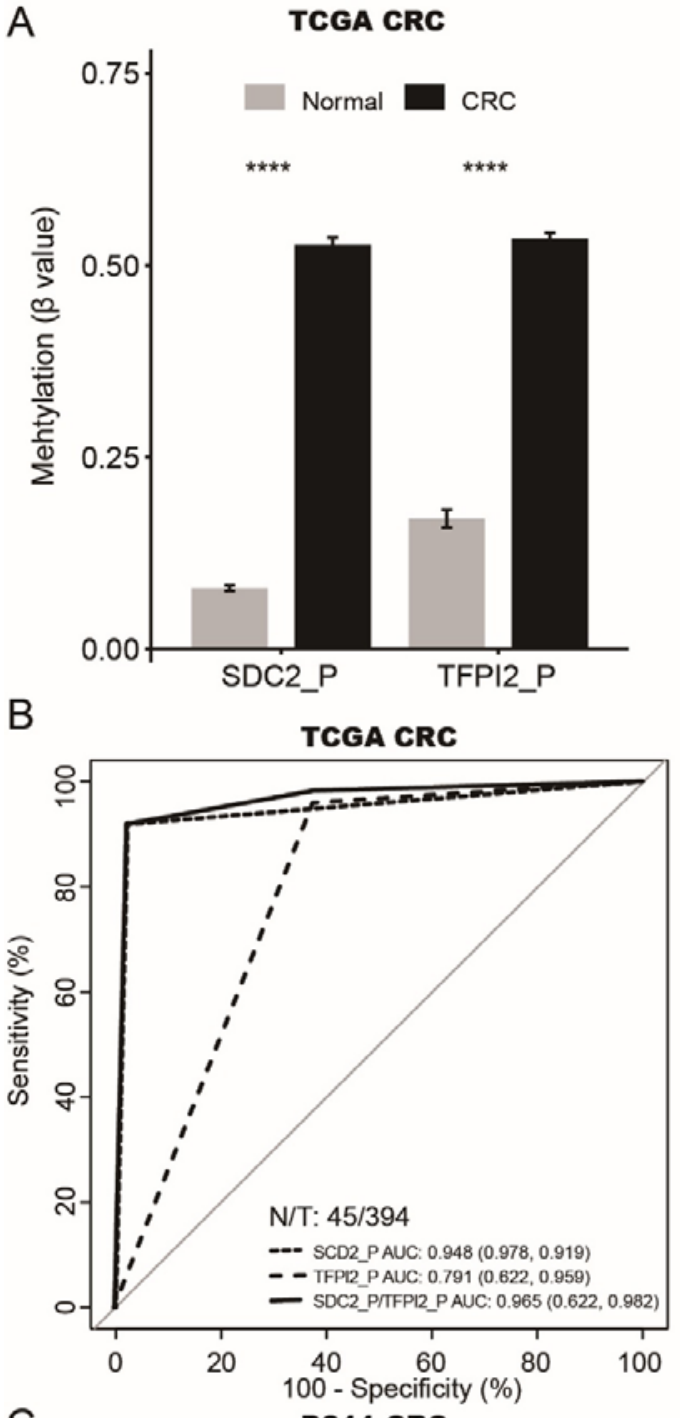

C

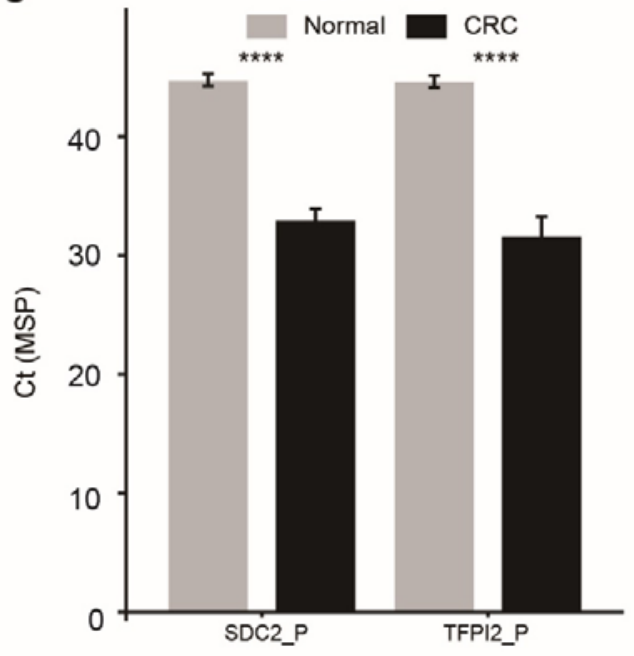

\section{GSE48684/GSE79740}
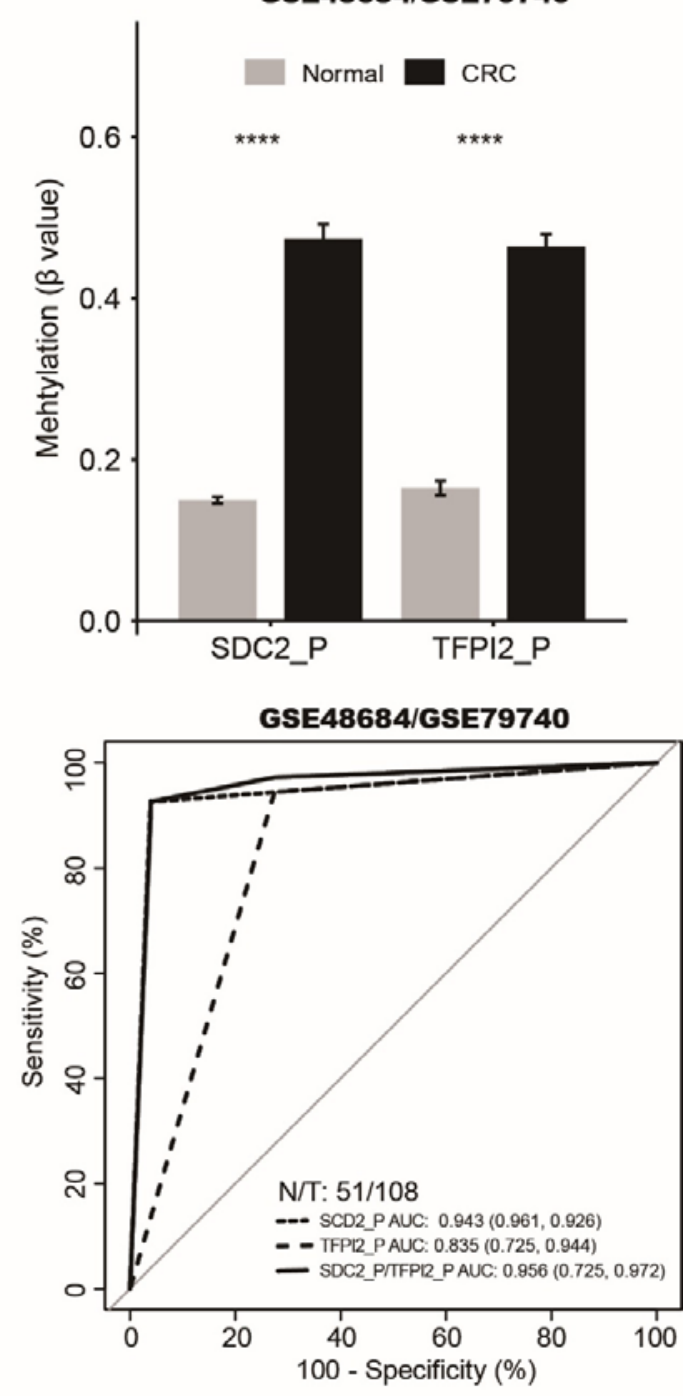

D311 CRC

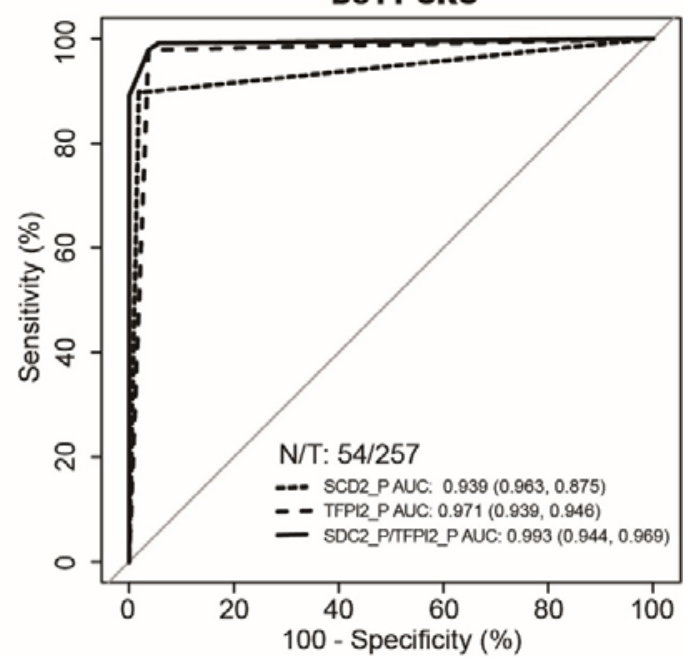

Figure 1 The performance of methylation levels of SDC2 and TFPI2 in distinguishing CRCs from normal controls。 $A: \beta$ values of SDC2 and TFPI 2 in $C R C s$ and normal controls。 $B$ : ROC curves and 
area under the curve to evaluate the performance of SDC2_P and TFPI2_P for distinguishing CRC

from normal samples. C : Ct values of SDC2 and TFPI2 in normal and CRC samples in D311 dataset。

Error bars represent mean $\beta$ value \pm sd.

A

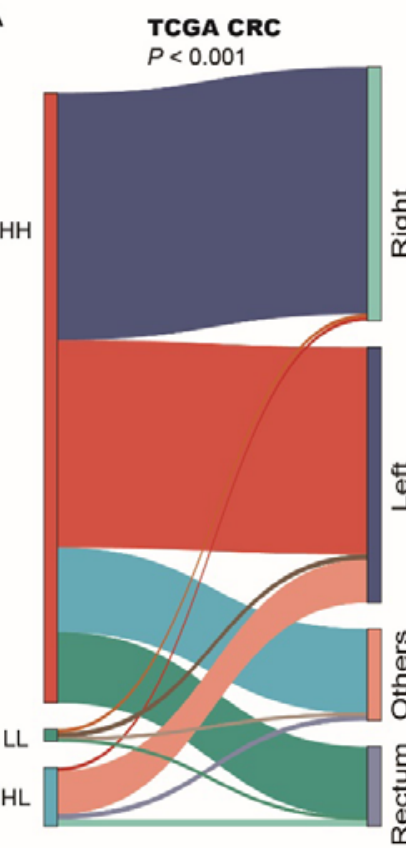

B

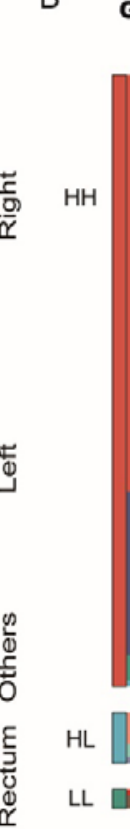

GSE48684/GSE79740 $P<0.05$

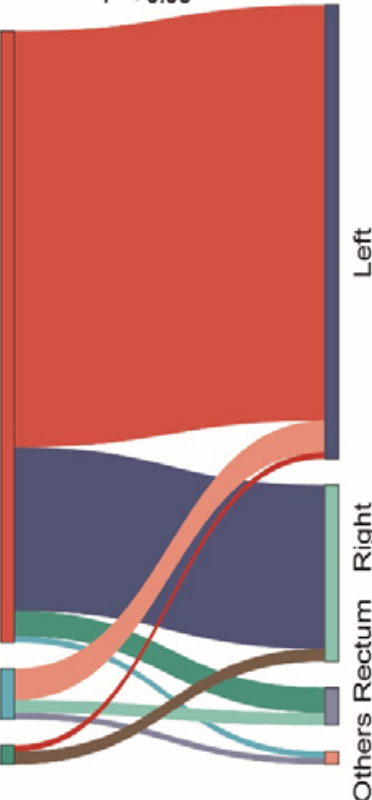

$\mathrm{C}$

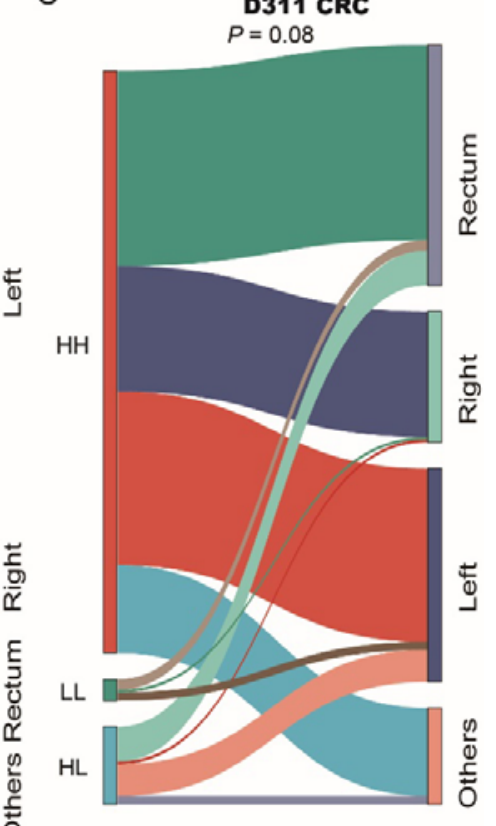

Figure 2 Sankey diagram showing the relationship of 3 methylator groups with tumor locations. Size of each rectangle and thickness of the connecting lines represents the number of samples on each group. Fisher exact test was used to calculate the $P$ value. 
A

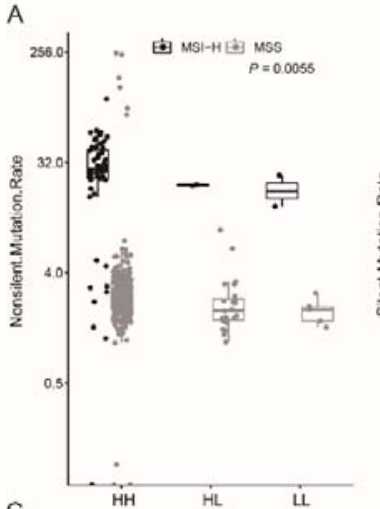

$\mathrm{C}_{854}$

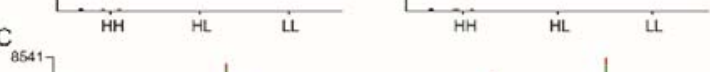

B
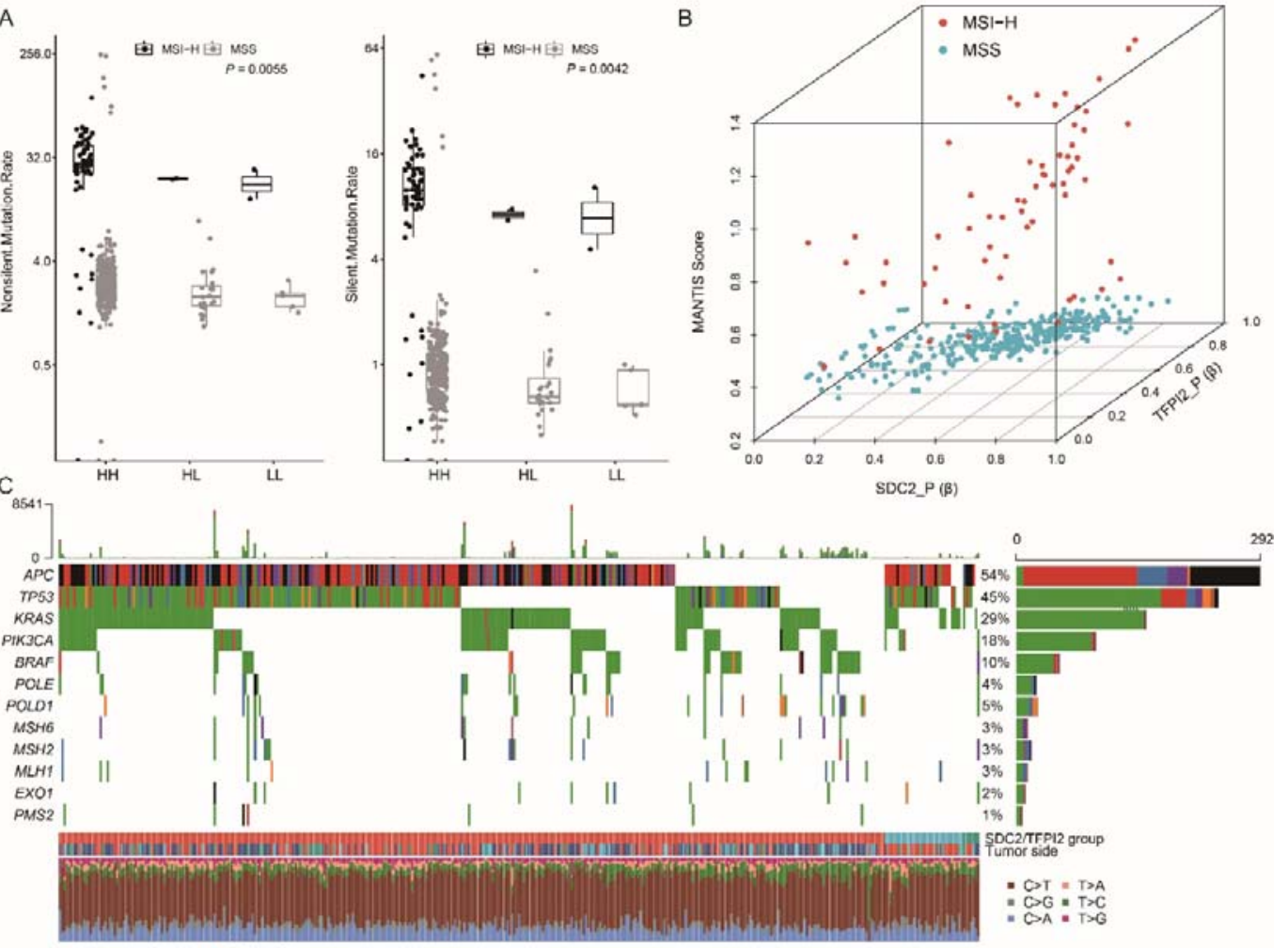

- Mrosense_Mutaton - Splice_Site DoubleTypeGroup ColonSite

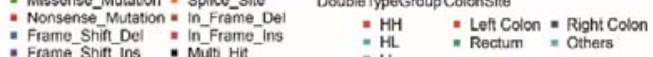

"Rectum "Others

D

TCGA CRC tumor location vs MSI

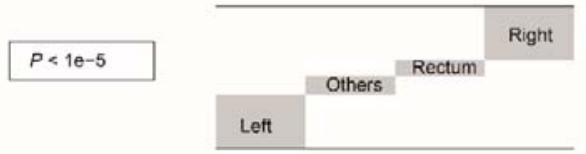

E
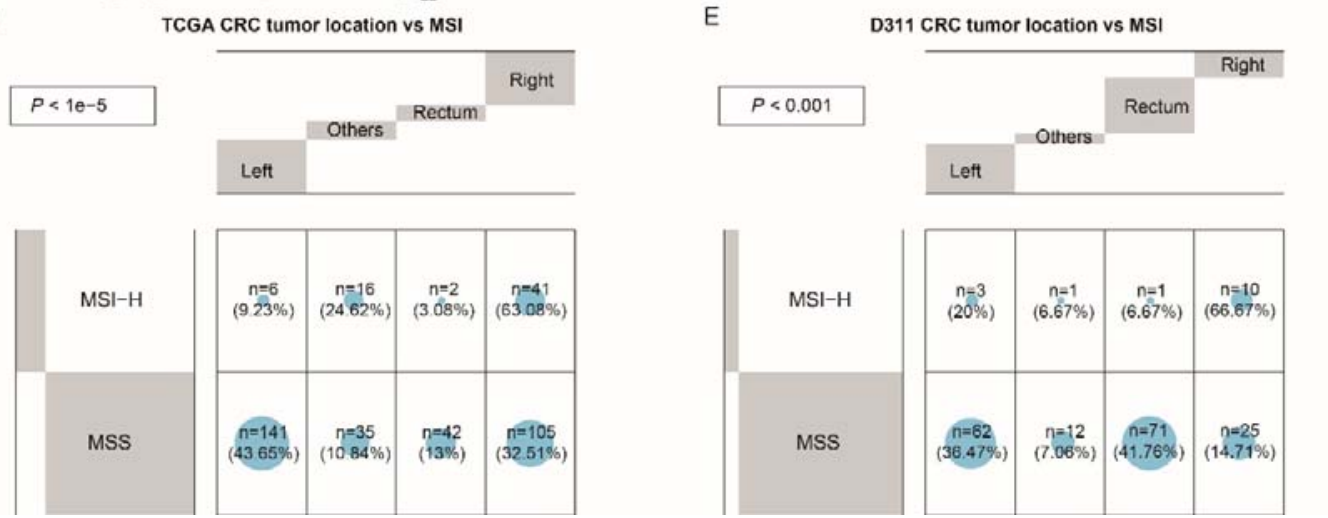

Figure 3 Genomic characteristics in three methylator groups. A: Nonsilent and silent mutation rates in $\mathrm{HH}, \mathrm{HL}$ and $\mathrm{LL}$ groups. B: The correlation of MANTIS score with $\beta$ values of SDC2 and TFPI2 in TCGA CRCs. C: Somatic mutation profile of five CIMP-related and MMR-related genes in HH, HL and LL groups. D\&E: Comparison of tumor location with MSI in TCGA CRCs (D) and D311 CRCs (E), significant $p$ value is calculated using fisher exact test. 
A

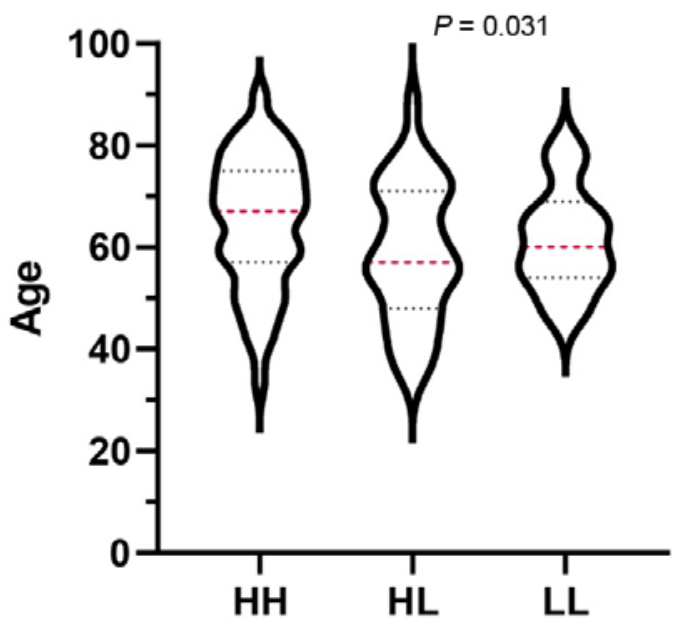

C

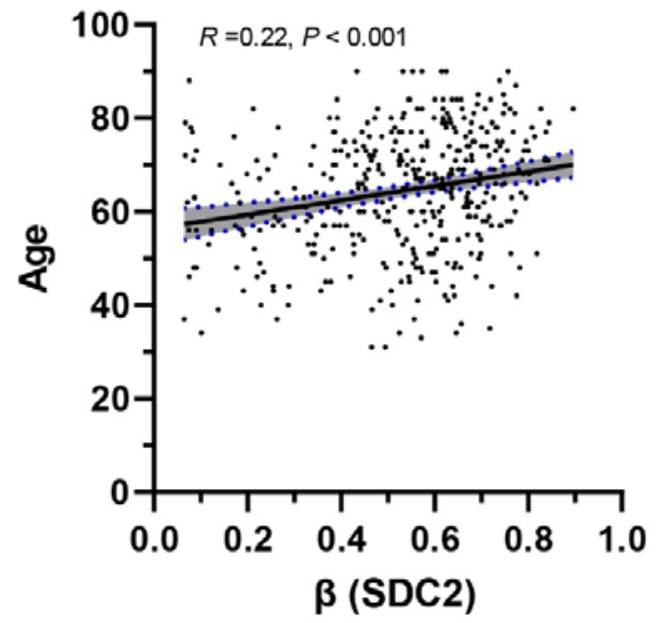

B

D311 CRC $(n=257)$

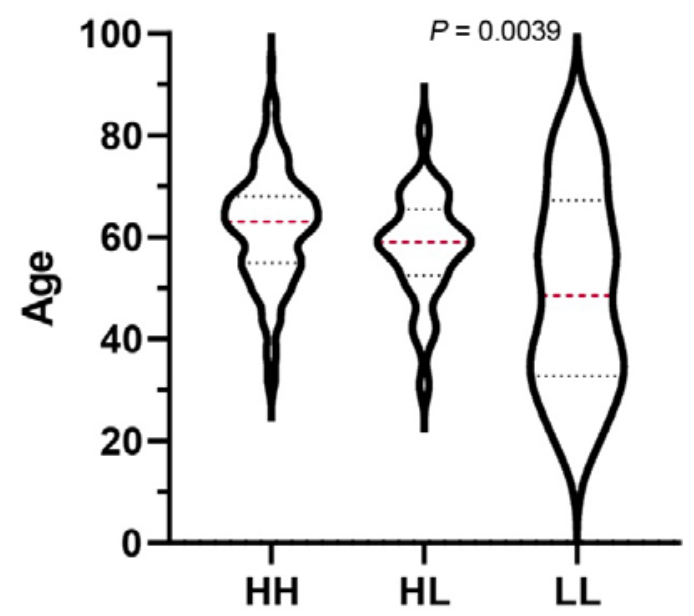

D

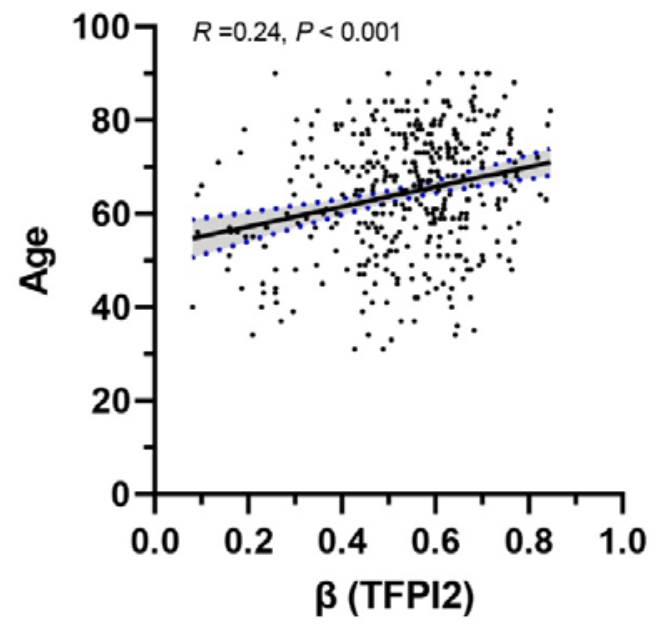

Figure 4 The association of patient age with methylation levels of SDC2 and TFPI2. A\&B: Patient ages in HH, HL and LL groups in TCGA CRCs (A) and D311 CRCs (B). The dashed red line indicate median age, while upper and lower dashed lines represent $75 \%$ quantile and $25 \%$ quantile. C\&D: The correlation of patient age with $\beta$ values of SDC2 and TFPI2 in TCGA CRCs. $P$ value and correlation coefficient was calculated using pearsion' method. 


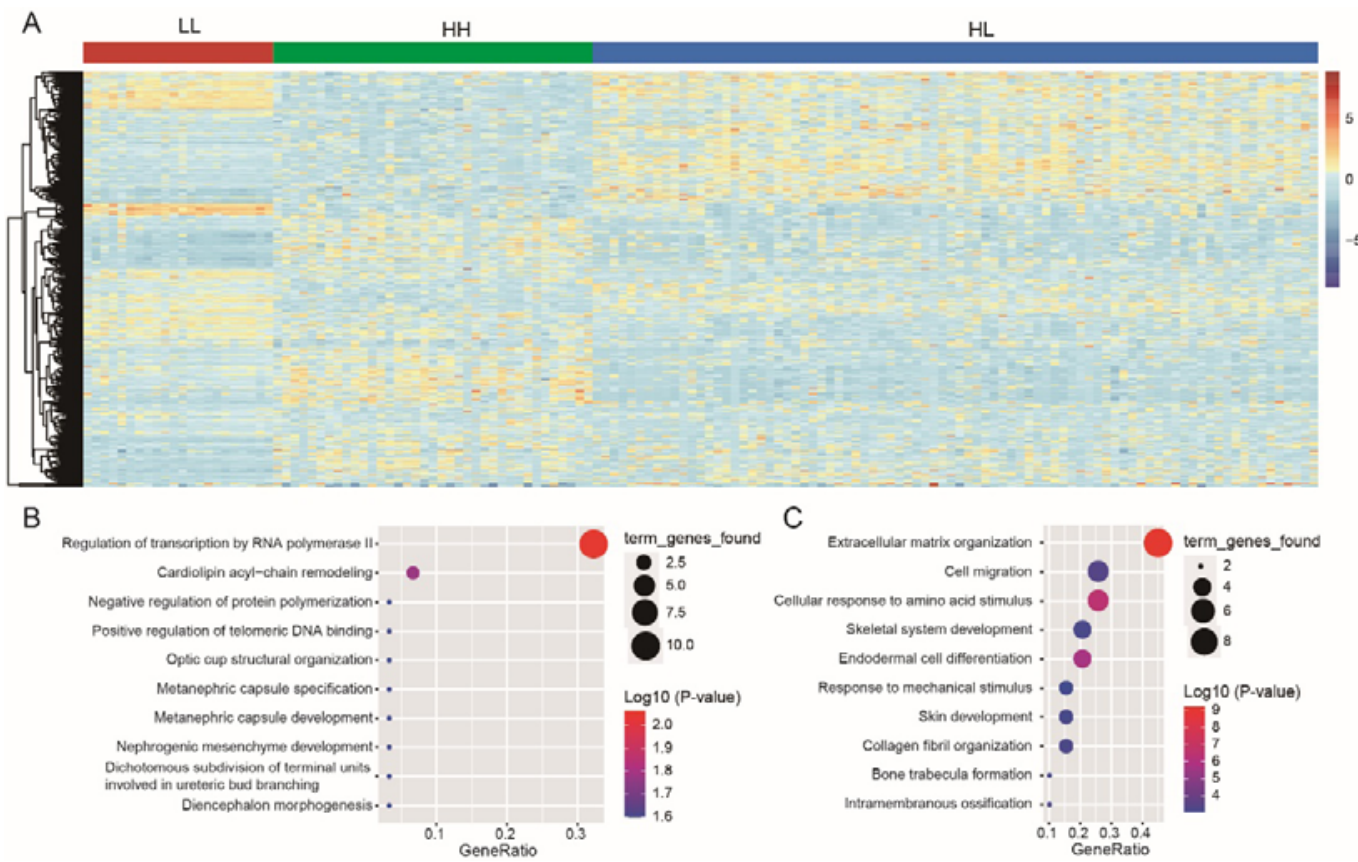

Figure 5 Identification of group specific DEGs. A: Expression heat map for the HH-, HL- and LL-specific DEGs. B: GO functional enrichment analyses for $\mathrm{HH}$-specific DEGs. C: GO functional enrichment analyses for LL-specific DEGs. The log10 (P-value) of each term is colored according to the legend.

\section{References}

1. Navarro, M.; Nicolas, A.; Ferrandez, A.; Lanas, A. Colorectal cancer population screening programs worldwide in 2016: An update. World J Gastroentero 2017, 23, 3632-3642, doi:10.3748/wjg.v23.i20.3632.

2. Ferlay, J.; Soerjomataram, I.; Dikshit, R.; Eser, S.; Mathers, C.; Rebelo, M.; Parkin, D.M.; Forman, D.; Bray, F. Cancer incidence and mortality worldwide: sources, methods and major patterns in GLOBOCAN 2012. Int J Cancer 2015, 136, E359-E386, doi:10.1002/ijc.29210.

3. Zhu, J.; Tan, Z.; Hollis-Hansen, K.; Zhang, Y.; Yu, C.; Li, Y. Epidemiological Trends in Colorectal Cancer in China: An Ecological Study. Digest Dis Sci 2017, 62, 235-243, doi:10.1007/s10620-016-4362-4.

4. Binefa, G.; Rodríguez-Moranta, F.; Teule, A.; Medina-Hayas, M. Colorectal cancer: from prevention to personalized medicine. World J Gastroenterol 2014, 20, 6786-6808, doi:10.3748/wjg.v20.i22.6786.

5. Ang, P.W.; Loh, M.; Liem, N.; Lim, P.L.; Grieu, F.; Vaithilingam, A.; Platell, C.; Yong, W.P.; lacopetta, B.; Soong, R. Comprehensive profiling of DNA methylation in colorectal cancer reveals subgroups with distinct clinicopathological and molecular features. Bmc Cancer 2010, 10, 227, doi:10.1186/1471-2407-10-227.

6. Azeem, S.; Gillani, S.W.; Siddiqui, A.; Jandrajupalli, S.B.; Poh, V.; Syed, S.S. Diet and Colorectal Cancer Risk in Asia--a Systematic Review. Asian Pac J Cancer Prev 2015, 16, 5389-5396, doi:10.7314/apjcp.2015.16.13.5389.

7. Gao, R.; Gao, Z.; Huang, L.; Qin, H. Gut microbiota and colorectal cancer. European journal 
of clinical microbiology \& infectious diseases : official publication of the European Society of Clinical Microbiology 2017, 36, 757-769, doi:10.1007/s10096-016-2881-8.

8. Gu, M.; Huang, Q.; Bao, C.; Li, Y.; Li, X.; Ye, D.; Ye, Z.; Chen, K.; Wang, J. Attributable causes of colorectal cancer in China. Bmc Cancer 2018, 18, 38, doi:10.1186/s12885-017-3968-z.

9. Boland, C.R.; Goel, A. Microsatellite instability in colorectal cancer. Gastroenterology 2010, 138, 2073-2087, doi:10.1053/j.gastro.2009.12.064.

10. Choi, S.; Choi, Y.; Jun, E.; Kim, I.S.; Kim, S.E.; Jung, S.A.; Oh, E.S. Shed syndecan-2 enhances tumorigenic activities of colon cancer cells. Oncotarget 2015, 6, 3874-3886, doi:10.18632/oncotarget.2885.

11. Park, H.; Kim, Y.; Lim, Y.; Han, I.; Oh, E.S. Syndecan-2 mediates adhesion and proliferation of colon carcinoma cells. J Biol Chem 2002, 277, 29730-29736, doi:10.1074/jbc.M202435200.

12. Vicente, C.M.; Ricci, R.; Nader, H.B.; Toma, L. Syndecan-2 is upregulated in colorectal cancer cells through interactions with extracellular matrix produced by stromal fibroblasts. Bmc Cell Bio/ 2013, 14, 25, doi:10.1186/1471-2121-14-25.

13. Hua, R.; Yu, J.; Yan, X.; Ni, Q.; Zhi, X.; Li, X.; Jiang, B.; Zhu, J. Syndecan-2 in colorectal cancer plays oncogenic role via epithelial-mesenchymal transition and MAPK pathway. Biomed Pharmacother 2020, 121, 109630, doi:https://doi.org/10.1016/j.biopha.2019.109630.

14. Sierko, E.; Wojtukiewicz, M.Z.; Kisiel, W. The role of tissue factor pathway inhibitor-2 in cancer biology. Semin Thromb Hemost 2007, 33, 653-659, doi:10.1055/s-2007-991532.

15. Chand, H.S.; Foster, D.C.; Kisiel, W. Structure, function and biology of tissue factor pathway inhibitor-2. Thromb Haemost 2005, 94, 1122-1130, doi:10.1160/TH05-07-0509.

16. Wang, G.; Huang, W.; Li, W.; Chen, S.; Chen, W.; Zhou, Y.; Peng, P.; Gu, W. TFPI-2 suppresses breast cancer cell proliferation and invasion through regulation of ERK signaling and interaction with actinin-4 and myosin-9. Sci Rep 2018, 8, 14402, doi:10.1038/s41598-018-32698-3.

17. Lavergne, M.; Jourdan, M.L.; Blechet, C.; Guyetant, S.; Pape, A.L.; Heuze-Vourc'H, N.; Courty, Y.; Lerondel, S.; Sobilo, J.; lochmann, S., et al. Beneficial role of overexpression of TFPI-2 on tumour progression in human small cell lung cancer. Febs Open Bio 2013, 3, 291-301, doi:10.1016/j.fob.2013.06.004.

18. Vaitkienè, P.; Skiriutè, D.; Skauminas, K.; Tamašauskas, A. Associations between TFPI-2 methylation and poor prognosis in glioblastomas. Medicina (Kaunas) 2012, 48, 345-349.

19. Xu, Y.; Qin, X.; Zhou, J.; Tu, Z.; Bi, X.; Li, W.; Fan, X.; Zhang, Y. Tissue factor pathway inhibitor- 2 inhibits the growth and invasion of hepatocellular carcinoma cells and is inactivated in human hepatocellular carcinoma. Oncol Lett 2011, 2, 779-783, doi:10.3892/ol.2011.340.

20. Oh, T.; Kim, N.; Moon, Y.; Kim, M.S.; Hoehn, B.D.; Park, C.H.; Kim, T.S.; Kim, N.K.; Chung, H.C.; An, S. Genome-wide identification and validation of a novel methylation biomarker, SDC2, for blood-based detection of colorectal cancer. J Mol Diagn 2013, 15, 498-507, doi:10.1016/j.jmoldx.2013.03.004.

21. Hibi, K.; Goto, T.; Kitamura, Y.H.; Yokomizo, K.; Sakuraba, K.; Shirahata, A.; Mizukami, H.; Saito, M.; Ishibashi, K.; Kigawa, G., et al. Methylation of TFPI2 gene is frequently detected in advanced well-differentiated colorectal cancer. Anticancer Res 2010, 30, 1205-1207.

22. Han, Y.D.; Oh, T.J.; Chung, T.; Jang, H.W.; Kim, Y.N.; An, S.; Kim, N.K. Early detection of colorectal cancer based on presence of methylated syndecan-2 (SDC2) in stool DNA. Clin 
Epigenetics 2019, 11, 51, doi:10.1186/s13148-019-0642-0.

23. Bagheri, H.; Mosallaei, M.; Bagherpour, B.; Khosravi, S.; Salehi, A.R.; Salehi, R. TFPI2 and NDRG4 gene promoter methylation analysis in peripheral blood mononuclear cells are novel epigenetic noninvasive biomarkers for colorectal cancer diagnosis. J Gene Med 2020, 22, e3189, doi:10.1002/jgm.3189.

24. Oh, T.J.; Oh, H.I.; Seo, Y.Y.; Jeong, D.; Kim, C.; Kang, H.W.; Han, Y.D.; Chung, H.C.; Kim, N.K.; An, S. Feasibility of quantifying SDC2 methylation in stool DNA for early detection of colorectal cancer. Clin Epigenetics 2017, 9, 126, doi:10.1186/s13148-017-0426-3.

25. Advani, S.M.; Advani, P.; DeSantis, S.M.; Brown, D.; VonVille, H.M.; Lam, M.; Loree, J.M.; Mehrvarz, S.A.; Bressler, J.; Lopez, D.S., et al. Clinical, Pathological, and Molecular Characteristics of $\mathrm{CpG}$ Island Methylator Phenotype in Colorectal Cancer: A Systematic Review and Meta-analysis. Transl Oncol 2018, 11, 1188-1201, doi:10.1016/j.tranon.2018.07.008.

26. Li, X.; Hu, F.; Wang, Y.; Yao, X.; Zhang, Z.; Wang, F.; Sun, G.; Cui, B.B.; Dong, X.; Zhao, Y. CpG island methylator phenotype and prognosis of colorectal cancer in Northeast China. Biomed Res Int 2014, 2014, 236361, doi:10.1155/2014/236361.

27. Missiaglia, E.; Jacobs, B.; D'Ario, G.; Di Narzo, A.F.; Soneson, C.; Budinska, E.; Popovici, V.; Vecchione, L.; Gerster, S.; Yan, P., et al. Distal and proximal colon cancers differ in terms of molecular, pathological, and clinical features. Ann Oncol 2014, 25, 1995-2001, doi:10.1093/annonc/mdu275.

28. Bufill, J.A. Colorectal cancer: evidence for distinct genetic categories based on proximal or distal tumor location. Ann Intern Med 1990, 113, 779-788, doi:10.7326/0003-4819-113-10-779.

29. Lee, M.S.; Menter, D.G.; Kopetz, S. Right Versus Left Colon Cancer Biology: Integrating the Consensus Molecular Subtypes. I Natl Compr Canc Netw 2017, 15, 411-419, doi:10.6004/jnccn.2017.0038.

30. Yamauchi, M.; Morikawa, T.; Kuchiba, A.; Imamura, Y.; Qian, Z.R.; Nishihara, R.; Liao, X.; Waldron, L.; Hoshida, Y.; Huttenhower, C., et al. Assessment of colorectal cancer molecular features along bowel subsites challenges the conception of distinct dichotomy of proximal versus distal colorectum. Gut 2012, 61, 847-854, doi:10.1136/gutjnl-2011-300865.

31. Yang, J.; Du XL; Li, S.T.; Wang, B.Y.; Wu, Y.Y.; Chen, Z.L.; Lv, M.; Shen, Y.W.; Wang, X.; Dong, D.F., et al. Characteristics of Differently Located Colorectal Cancers Support Proximal and Distal Classification: A Population-Based Study of 57,847 Patients. Plos One 2016, 11, e167540, doi:10.1371/journal.pone.0167540.

32. Chen, J.; Sun, H.; Tang, W.; Zhou, L.; Xie, X.; Qu, Z.; Chen, M.; Wang, S.; Yang, T.; Dai, Y., et al. DNA methylation biomarkers in stool for early screening of colorectal cancer. J Cancer 2019, 10, 5264-5271, doi:10.7150/jca.34944.

33. Sun, M.; Liu, J.; Hu, H.; Guo, P.; Shan, Z.; Yang, H.; Wang, J.; Xiao, W.; Zhou, X. A novel panel of stool-based DNA biomarkers for early screening of colorectal neoplasms in a Chinese population. I Cancer Res Clin Oncol 2019, 145, 2423-2432, doi:10.1007/s00432-019-02992-2.

34. Zhao, G.; Li, H.; Yang, Z.; Wang, Z.; Xu, M.; Xiong, S.; Li, S.; Wu, X.; Liu, X.; Wang, Z., et al. Multiplex methylated DNA testing in plasma with high sensitivity and specificity for colorectal cancer screening. Cancer Med 2019, 8, 5619-5628, doi:10.1002/cam4.2475. 
35. Colaprico, A.; Silva, T.C.; Olsen, C.; Garofano, L.; Cava, C.; Garolini, D.; Sabedot, T.S.; Malta, T.M.; Pagnotta, S.M.; Castiglioni, I., et al. TCGAbiolinks: an R/Bioconductor package for integrative analysis of TCGA data. Nucleic Acids Res 2016, 44, e71, doi:10.1093/nar/gkv1507.

36. Luo, Y.; Wong, C.J.; Kaz, A.M.; Dzieciatkowski, S.; Carter, K.T.; Morris, S.M.; Wang, J.; Willis, J.E.; Makar, K.W.; Ulrich, C.M., et al. Differences in DNA methylation signatures reveal multiple pathways of progression from adenoma to colorectal cancer. Gastroenterology 2014, 147, 418-429, doi:10.1053/j.gastro.2014.04.039.

37. Alvi, M.A.; Loughrey, M.B.; Dunne, P.; McQuaid, S.; Turkington, R.; Fuchs, M.A.; McGready, C.; Bingham, V.; Pang, B.; Moore, W., et al. Molecular profiling of signet ring cell colorectal cancer provides a strong rationale for genomic targeted and immune checkpoint inhibitor therapies. Br J Cancer 2017, 117, 203-209, doi:10.1038/bjc.2017.168.

38. Sun, Z.; Chai, H.S.; Wu, Y.; White, W.M.; Donkena, K.V.; Klein, C.J.; Garovic, V.D.; Therneau, T.M.; Kocher, J.P. Batch effect correction for genome-wide methylation data with Illumina Infinium platform. Bmc Med Genomics 2011, 4, 84, doi:10.1186/1755-8794-4-84.

39. Franzin, A.; Sambo, F.; Di Camillo, B. bnstruct: an R package for Bayesian Network structure learning in the presence of missing data. Bioinformatics 2017, 33, 1250-1252, doi:10.1093/bioinformatics/btw807.

40. Gervaz, P.; Usel, M.; Rapiti, E.; Chappuis, P.; Neyroud-Kaspar, I.; Bouchardy, C. Right colon cancer: Left behind. Eur J Surg Oncol 2016, 42, 1343-1349, doi:10.1016/j. ejso.2016.04.002.

41. Ooki, A.; Maleki, Z.; Tsay, J.J.; Goparaju, C.; Brait, M.; Turaga, N.; Nam, H.S.; Rom, W.N.; Pass, H.I.; Sidransky, D., et al. A Panel of Novel Detection and Prognostic Methylated DNA Markers in Primary Non-Small Cell Lung Cancer and Serum DNA. Clin Cancer Res 2017, 23, 7141-7152, doi:10.1158/1078-0432.CCR-17-1222.

42. Love, M.I.; Huber, W.; Anders, S. Moderated estimation of fold change and dispersion for RNA-seq data with DESeq2. Genome Biol 2014, 15, 550, doi:10.1186/s13059-014-0550-8.

43. Tabas-Madrid, D.; Nogales-Cadenas, R.; Pascual-Montano, A. GeneCodis3: a non-redundant and modular enrichment analysis tool for functional genomics. Nucleic Acids Res 2012, 40, W478-W483, doi:10.1093/nar/gks402.

44. Du, P.; Zhang, X.; Huang, C.; Jafari, N.; Kibbe, W.A.; Hou, L.; Lin, S.M. Comparison of Beta-value and $M$-value methods for quantifying methylation levels by microarray analysis. Bmc Bioinformatics 2010, 11, 587, doi:10.1186/1471-2105-11-587.

45. Ma, X.; Wang, Y.W.; Zhang, M.Q.; Gazdar, A.F. DNA methylation data analysis and its application to cancer research. Epigenomics-Uk 2013, 5, 301-316, doi:10.2217/epi.13.26.

46. Kautto, E.A.; Bonneville, R.; Miya, J.; Yu, L.; Krook, M.A.; Reeser, J.W.; Roychowdhury, S. Performance evaluation for rapid detection of pan-cancer microsatellite instability with MANTIS. Oncotarget 2017, 8, 7452-7463, doi:10.18632/oncotarget.13918.

47. Hibi, K.; Goto, T.; Shirahata, A.; Saito, M.; Kigawa, G.; Nemoto, H.; Sanada, Y. Detection of TFPI2 methylation in the serum of colorectal cancer patients. Cancer Lett 2011, 311, 96-100, doi:10.1016/j.canlet.2011.07.006.

48. Imperiale, T.F.; Ransohoff, D.F.; Itzkowitz, S.H.; Levin, T.R.; Lavin, P.; Lidgard, G.P.; Ahlquist, D.A.; Berger, B.M. Multitarget stool DNA testing for colorectal-cancer screening. $N$ Engl J Med 2014, 370, 1287-1297, doi:10.1056/NEJMoa1311194.

49. Yang, H.; Xia, B.Q.; Jiang, B.; Wang, G.; Yang, Y.P.; Chen, H.; Li, B.S.; Xu, A.G.; Huang, Y.B.; 

available under aCC-BY-NC-ND 4.0 International license.

Wang, X.Y. Diagnostic value of stool DNA testing for multiple markers of colorectal cancer and advanced adenoma: a meta-analysis. Can J Gastroenterol 2013, 27, 467-475, doi:10.1155/2013/258030.

50. Zong, L.; Abe, M.; Ji, J.; Zhu, W.G.; Yu, D. Tracking the Correlation Between CpG Island Methylator Phenotype and Other Molecular Features and Clinicopathological Features in Human Colorectal Cancers: A Systematic Review and Meta-Analysis. Clin Transl Gastroenterol 2016, 7, e151, doi:10.1038/ctg.2016.14.

51. Xian, X.; Gopal, S.; Couchman, J.R. Syndecans as receptors and organizers of the extracellular matrix. Cell Tissue Res 2010, 339, 31-46, doi:10.1007/s00441-009-0829-3. 